\title{
New results of investigations of whistler-mode chorus emissions
}

\author{
O. Santolík \\ Institute of Atmospheric Physics, Prague and Charles University, Prague, Czech Republic \\ Received: 4 April 2008 - Revised: 24 June 2008 - Accepted: 3 July 2008 - Published: 30 July 2008
}

\begin{abstract}
This review summarizes selected recent results obtained during investigation of whistler-mode chorus emissions in the Earth's magnetosphere. Special attention is paid to results published during the last five years, with a focus on the results of the CLUSTER project. The nonlinear nature of chorus emissions is demonstrated using both theoretical results and measurements. Selected areas of research on whistler-mode chorus are covered and the paper especially reports new results on substructure and amplitudes of chorus wave packets, on new observations of frequency differences of chorus wave packets at different points in space and on their possible interpretations, on results concerning determination of position and size of the source region of chorus, on recent observational and theoretical results which lead to improved description of propagation of chorus from its source, and, finally, on comparison of chorus measurements with corresponding values deduced from nonlinear theory and simulations.
\end{abstract}

\section{Introduction}

Chorus is one of the most intense natural wave emissions generated by plasma instabilities in the Earth's magnetosphere, observed first on the ground in the local morning hours (Storey, 1953; Helliwell, 1969). It occurs in the frequency range from a few hundreds of Hertz to several kHz, with complex frequency spectra often containing many distinct nonlinear wave packets with changing frequency at time scales of a fraction of a second. These features resemble the sound of chirping birds in the morning, from where came the original name of "dawn chorus". As first described by Storey

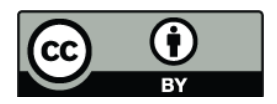

Correspondence to: O. Santolík (ondrej.santolik@mff.cuni.cz)
(1953): "The sound of the 'dawn chorus' may be likened to that of a rookery heard from a distance."

The generation mechanism of chorus has been extensively investigated in the past (see reviews by Sazhin and Hayakawa, 1992; Omura et al., 1991). It is now widely accepted that chorus is generated by a nonlinear process (e.g. Nunn et al., 1997; Trakhtengerts, 1999) which involves the electron cyclotron resonance of whistler-mode waves with energetic electrons in the Earth's magnetosphere (Andronov and Trakhtengerts, 1964; Kennel and Petschek, 1966). However, important properties of the generation mechanism of chorus are not yet well understood.

Early satellite observations showed that chorus is usually observed outside of the plasmasphere (Gurnett and O'Brien, 1964). Spacecraft measurements also confirmed the predominant occurrence of chorus during the local morning and day time (e.g. Burtis and Helliwell, 1976; Cornilleau-Wehrlin et al., 1978; Koons and Roeder, 1990). This can be explained by the drift of the source population of electrons in the Earth's magnetic field from the magnetospheric tail earthward and toward the local morning.

Generation of a large class of chorus events takes place close to the geomagnetic equatorial plane (Burton and Holzer, 1974; Tsurutani and Smith, 1977; LeDocq et al., 1998; Lauben et al., 2002), where the first derivative of the magnetic field strength along a geomagnetic field line is close to zero (Helliwell, 1967). In the source region, two frequency bands of chorus have been observed (Tsurutani and Smith, 1974; Burtis and Helliwell, 1976; Goldstein and Tsurutani, 1984), below and above one half of the local electron cyclotron frequency. These bands are separated by a narrow frequency interval of decreased intensity just below one half of the local electron cyclotron frequency. Emissions of equatorial chorus are often observed during periods of disturbed magnetospheric conditions (Tsurutani and Smith, 1974, 1977; Inan et al., 1992; Lauben et al., 1998; Meredith et al., 2001), in association with anisotropic

Published by Copernicus Publications on behalf of the European Geosciences Union and the American Geophysical Union. 
electrons at energies of 10-100 keV (Burton, 1976; Anderson and Maeda, 1977)

Although chorus has been known for more than 50 years, it recently regained an increased attention in connection with the research on acceleration mechanisms of energetic electrons (Summers et al., 1998; Horne and Thorne, 1998). Whistler-mode chorus is being considered as a local source of highly accelerated electrons in the outer Van Allen radiation belt (Meredith et al., 2001, 2003a; Horne and Thorne, 2003; Horne et al., 2003; Horne et al., 2005; Demekhov et al., 2006; Chen et al., 2007; Horne, 2007). These electrons can cause serious damage to spacecraft systems, and, if precipitated into the upper atmosphere, they can influence the atmospheric composition. Investigation of whistler-mode chorus could thus not only contribute to a basic understanding of our space environment, but also it could potentially have some practical consequences.

This review paper summarizes recent results obtained during investigation of whistler-mode chorus. The main focus is on selected experimental results published during the last five years, with a special attention to the results of the CLUSTER project and to results which demonstrate the nonlinear nature of chorus emissions. Sections 2 and 3 report on new results on amplitudes and fine structure of chorus wave packets, Sect. 4 presents new observations of frequency differences of chorus wave packets in different points in space and their interpretations, Sect. 5 refers to recent results on position and size of the source region of chorus, Sect. 6 summarizes observational and theoretical results on propagation of chorus from its source, and, finally, Sect. 7 reports on comparison of chorus measurements with corresponding values deduced from nonlinear theory and computer simulations.

\section{Expected amplitudes of nonlinear chorus wave packets}

Linear and quasi-linear approaches are often effectively used as a useful approximation for properties of wave-particle interactions connected with chorus, especially for the purpose of global modeling of radiation belt dynamics (e.g. Horne et al., 2005; Shprits et al., 2006, 2007; Summers et al., 2007a,b; Bortnik et al., 2008; Albert, 2008, and references therein). However, the discrete structure of chorus wave packets strongly suggests that nonlinear effects are important for the microphysics of wave particle interactions (Omura et al., 1991). Larger wave amplitudes could imply strong nonlinear effects (Nunn et al., 1997; Trakhtengerts, 1999) leading to an instability with a faster growth compared to the linear approximation.

Omura and Summers (2004) showed, using electromagnetic particle simulations in a simplified model without including the spatial inhomogeneity of the terrestrial magnetic field $B_{0}$, that whistler mode waves grow nonlinearly after they reached amplitudes of $\approx 3 \times 10^{-3} B_{0}$. The nonlinear saturation level which occurred at amplitudes of $\approx 2 \times 10^{-2} B_{0}$ due to a combination of nonlinear trapping of resonant electrons and quasi-linear relaxation of the temperature anisotropy of the source population of relativistic electrons. Katoh and Omura $(2006,2007)$ recently published results of an extensive simulation study using the electron hybrid model, where they included a strong spatial inhomogeneity of $B_{0}$. They reported maximum amplitudes of chorus wave packets of the order of $10^{-3} B_{0}$ and important nonlinear currents contributing to wave growth and frequency drift.

Omura and Summers (2006) found important nonlinear trapping of resonant particles at lower wave amplitudes of $\approx 10^{-4} B_{0}$. This effect then leads to the formation of an electromagnetic electron hole in the velocity space. Omura et al. (2007) then showed that wave amplitudes of $\approx 6 \times 10^{-6}$ $6 \times 10^{-4} B_{0}$ are sufficient for resonant trapping and acceleration of relativistic electrons to take place at different realistic distances from the geomagnetic equator. This "relativistic turning acceleration" then can lead to the energy increase whose maximum value is proportional to the wave amplitude of sufficiently long wave packets $(\approx 1 \mathrm{~s})$ of constant frequency.

Nunn et al. (1997) used the Vlasov-hybrid simulation (VHS) method to reproduce observations of chorus wave packets by the Geotail spacecraft in the dayside magnetosphere. They found strong nonlinear currents and wave growth already for amplitudes of the order of $10^{-5} B_{0}$, with the saturation level at $1.6 \times 10^{-3} B_{0}$ for $B_{0} \approx 100 \mathrm{nT}$. Nunn et al. (2005) used an improved VHS method (Nunn et al., 2003) for a simulation of whistler-mode wave packets triggered by a weak signal of $3 \times 10^{-7} B_{0}$ for the magnetic field model at Mcllwain's parameter $L=4.4$ (equatorial dipole $B_{0} \approx 350 \mathrm{nT}$ ), and in a dense plasma with a number density $n_{e}=400 \mathrm{~cm}^{-3}$. They again found that already a wave amplitude of $2 \times 10^{-5} B_{0}$ gave a strongly nonlinear situation, with many trapping lengths fitting into the generation region of a wave packet with slowly rising frequency.

All the above mentioned theoretical studies supposed that the whistler-mode waves propagate with wave vectors close to the $\mathbf{B}_{0}$ direction. Bell $(1984,1986)$ derived conditions of nonlinear trapping for waves with arbitrary wave-vector directions. He predicted that whistler-mode waves can, during their propagation through the magnetosphere, encounter one or several regions where the wave amplitudes required for trapping are minimum. The minimum amplitudes were again of the order of $10^{-5}-10^{-4} B_{0}$ at $L$ between 4 and 5 .

\section{Observed amplitudes and fine structure of chorus wave packets}

Now, the question arises if these amplitudes (of the order of $10^{-4} B_{0}$, or 20 to $50 \mathrm{pT}$ at $L=4-5$ ) can really be observed in the naturally generated emissions of whistler-mode chorus. 
The results of Cornilleau-Wehrlin et al. (1976) indicated that saturation amplitudes of chorus wave packets can reach this limit. Further systematic observations confirmed that in most cases the amplitudes of chorus emissions are indeed sufficiently high to be governed by nonlinear effects.

Meredith et al. (2001) analyzed electric field measurements of the CRRES spacecraft and observed equatorial chorus with average amplitudes $E_{w}$ of the order of $1 \mathrm{mV} / \mathrm{m}$ during geomagnetic substorms. The plasma frequency $f_{p}$ has been estimated for these conditions from wave measurements, yielding the ratio to the electron cyclotron frequency $f_{p} / f_{c}$ between 1 and 5 (Meredith et al., 2002). This, using the cold plasma theory in the frequency range of lower-band chorus $\left(f=0.1-0.5 f_{c}\right)$, gives the refractive index of parallel propagating whistler-mode waves,

$N=\sqrt{1+\frac{\left(f_{p} / f_{c}\right)^{2}}{\frac{f}{f_{c}}\left(1-\frac{f}{f_{c}}\right)}}$,

yielding $N$ between 2 and 16 . The magnetic-field amplitudes of chorus can be then estimated from the Faraday's law, $B_{w}=E_{w} N / c$, supposing again propagation parallel to the magnetic field lines ( $c$ is the light speed). We thus obtain average $B_{w}$ of the order of 10-100 pT, corresponding also to results of Meredith et al. (2003b) where similar analysis of averaged amplitudes of whistler-mode waves is done for different levels of geomagnetic activity. However, the time resolution of these measurements is insufficient to resolve the separate wave packets of chorus and average amplitudes are calculated over time intervals corresponding to many characteristic durations of chorus wave packets, assuming their presence.

Investigation of the amplitudes of separate wave packets of chorus requires high-resolution measurements of wave electric or magnetic fields, such as those recorded by the Wideband data (WBD) instrument (Gurnett et al., 1997, 2001) onboard the CLUSTER spacecraft (Escoubet et al., 1997). Fine structure of chorus waveforms has been investigated using measurements obtained during geomagnetic storms (Santolík et al., 2003, 2004b). Analyzed wave packets appeared as rising discrete elements of lower-band chorus with the frequency drift of $10-20 \mathrm{kHz} / \mathrm{s}$. The waveform of the measured electric field has shown an internal fine structure of each wave packet consisting of a sequence of separate subpackets. This effect could be explained by generation of frequency sidebands during the evolution of a chorus wave packet and by the beating effect of simultaneously present signals at closely separated frequencies (Nunn et al., 2005).

Initial analysis of this fine structure has been done using a sine-wave parametric model with a variable amplitude (Santolík et al., 2003). Some subpackets start with an exponential growth phase, and after reaching the saturation amplitude they can also sometimes show an exponential decay phase. The duration of subpackets is variable from a few milliseconds to a few tens of milliseconds (see Fig. 1). The growth

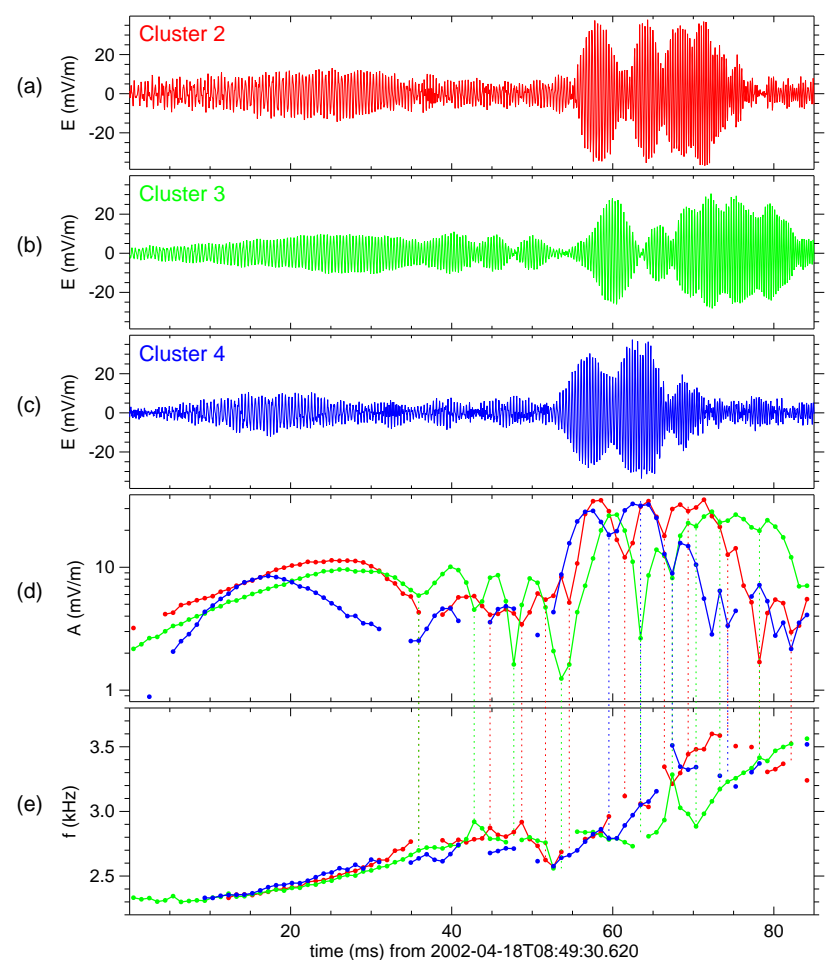

Fig. 1. Waveforms and model parameters in an 85-ms time interval covering a chorus element recorded by the WBD instrument onboard CLUSTER during geomagnetically disturbed conditions on 18 April 2002. (a-c) Broadband electric field waveform for CLUSTER 2-4, respectively; (d) Amplitude; (e) Frequency. The results from the three spacecraft are color-coded. In order to demonstrate the fine structure, minima of amplitude are connected with the corresponding frequency estimates by vertical dotted lines (from Santolík et al., 2003).

rate of the initial linear phase can be highly variable from case to case. Typical values of imaginary part of the wave frequency thus vary between a few tens and a few hundreds of $\mathrm{s}^{-1}$. The detected amplitudes of the subpackets reached more than $30 \mathrm{mV} / \mathrm{m}$. Using a preliminary estimate of $f_{p} / f_{c} \approx 1.5$ from the local plasma density measurements by the WHISPER instrument (Décréau et al., 2001), these amplitudes approximately correspond to $300 \mathrm{pT}\left(\approx 10^{-3} B_{0}\right)$ for the magnetic component of chorus. Improved analysis (P. Décréau, private communication, 2007) gives $f_{p} / f_{c} \approx 2.4$ and a somewhat higher estimate for magnetic amplitudes of $\approx 500 \mathrm{pT}$. Note, however, that the instrumental upper limit for amplitudes in that study was $\approx 39 \mathrm{mV} / \mathrm{m}$ and that many observed waveforms had peaks above that limit and could not be used in the analysis. Analysis of a large number of chorus wave packets (Santolík et al., 2004b) showed that the typical delay between the two neighboring maxima of the wave amplitude is a few milliseconds with a decreasing probability density toward longer delays. The subpackets with largest amplitudes were found embedded in the interior of the separate 
wave packets. This study also indicates that the highest amplitudes are rare and that most common amplitudes in the examined case ranged between a few $\mathrm{mV} / \mathrm{m}$ to about $10 \mathrm{mV} / \mathrm{m}$, corresponding to a few hundreds of $\mathrm{pT}\left(\approx 10^{-3} B_{0}\right)$.

Cattell et al. (2008) recently reported whistler-mode waves in the dawn-side outer radiation belt, with amplitudes up to the upper limit of detection $(240 \mathrm{mV} / \mathrm{m})$ of the S/WAVES instruments onboard the STEREO spacecraft. This is 6 times higher than the upper limit for measurement of electric field amplitudes by the WBD instrument onboard CLUSTER but, on the other hand, only waveforms with largest amplitudes were recorded on Stereo. A proper survey of wave amplitudes thus could not be done and, based on previous CLUSTER results, it is probable that these high amplitudes are rare. Note that Cattell et al. (2008) did not interpret their observations as whistler-mode chorus although many observed characteristics are similar.

All these new results on amplitudes and fine structure of wave packets of chorus or whistler mode waves in general strongly suggest that nonlinear effects play an important role in microphysics governing the interactions of these waves with particle populations, including the energetic electrons from the outer Van Allen radiation belt.

\section{Frequency differences of chorus wave packets ob- served by different spacecraft}

Gurnett et al. (2001) first reported unexpected frequency differences of $\approx 1 \mathrm{kHz}$ between nearly identical discrete wave packets that were observed simultaneously by different CLUSTER spacecraft.

Inan et al. (2004) and Platino et al. (2006) interpreted these observations by differential Doppler shift from rapidly moving elementary sources at speeds comparable to the parallel velocity of counter-streaming resonant electrons. This interpretation is based on the dependence of the whistler-mode refractive index on the angle between the wave vector and the static magnetic field and on the assumption of rapid motion of highly localized source regions of chorus moving at large speeds of thousands to tens of thousands $\mathrm{km} / \mathrm{s}$. Waves from the localized sources propagate to different spacecraft at different wave normal angles, and thus at different phase and group speeds and, as a consequence, the differential Doppler shift and time delay between the two spacecraft occurs. This results in differences in observed frequency, as well as the different times of arrival of the similar emissions at the different spacecraft. However, a realistic plasma physical interpretation of rapid motions of chorus source regions needs to be found.

Breneman et al. (2007) and Chum et al. (2007) proposed an alternative explanation based on their ray-tracing simulations of waves with different frequencies propagating from a localized source to different CLUSTER spacecraft. These different frequencies can compose a single chorus element in the source from which each spacecraft can only receive a limited frequency interval. Breneman et al. (2007) have shown that some of the observed time and frequency shifts can result from limited accessibility of the spacecraft positions for whistler-mode waves emitted from a stationary source located close to the equatorial plane. In this paper, the position of the source region has been found using a reverse ray-tracing technique started from the observation points. Chum et al. (2007) tested the hypothesis of a source which emits waves in a relatively narrow interval of wave normal angles and that varies both the wave normal angle and frequency during the generation of a single chorus element. They demonstrated that the forward ray tracing simulation leads to patterns which are very similar to those observed. They also succeeded to simulate events with large time shifts between the corresponding elements observed on different spacecraft.

Several possible explanations thus exist at present and these theories wait for future experimental tests to be done.

\section{Position and size of the chorus source region}

Multi-point measurements of the CLUSTER mission resulted also in determination of the characteristic size of the sources of separate wave packets of chorus. For a favorable case when the separation distance of the CLUSTER spacecraft was only of the order of hundreds of $\mathrm{km}$, chorus emissions were observed in their generation region close to the magnetic equatorial plane at a radial distance of 4.4 Earth's radii. Different spacecraft observed very similar wave packets of lower-band chorus below one half of the electron cyclotron frequency. Both linear and rank correlation analysis have been used (Santolík and Gurnett, 2003; Santolík et al., 2004a) to define perpendicular dimensions of the sources of these wave packets. Correlation was significant in the range of separation distances up to $260 \mathrm{~km}$ parallel to the field line and up to $100 \mathrm{~km}$ in the perpendicular plane. In this range, the correlation coefficient was independent of parallel separations, and decreased as a function of perpendicular separation. The characteristic perpendicular scale for decrease of the correlation coefficient varied between 60 and $200 \mathrm{~km}$ for different time intervals when the spacecraft measured inside the source region. This variation was consistent with the expected effect of random positions of locations at which the individual chorus wave packets were generated. Statistical properties of the observations were consistent with a model of the source region consisting of individual independent random sources emitting quasi-periodic series of wave packets. In this model, the wave power was radiated from the individual active areas with gaussian two-dimensional profiles having a common half-width of $35 \mathrm{~km}$ in the plane perpendicular to the magnetic field (Santolík et al., 2004a). This characteristic scale was comparable to the wavelength of the observed whistler-mode waves. 
Multi-point measurement of the Poynting flux by the STAFF-SA instruments (Cornilleau-Wehrlin et al., 2003) onboard CLUSTER was used to investigate the position of the chorus source region (Parrot et al., 2003a; Santolík et al., 2004b, 2005a). Observed spatio-temporal variations of the direction of the Poynting flux consistently showed that the central position of the chorus source fluctuates on time scales of minutes within $1000-2000 \mathrm{~km}$ of the geomagnetic equatorial plane. Estimates of the electromagnetic planarity were used to characterize the extent of the source region in the direction parallel to the field line, obtaining a range of 3000$5000 \mathrm{~km}$ (Santolík et al., 2004b, 2005a). The typical order of magnitude of the global speed of motion of the entire source region was $100 \mathrm{~km} / \mathrm{s}$, as determined from average propagation properties of several neighboring chorus wave packets.

Concerning the global extent of the chorus distribution in the plane of geomagnetic equator, new results have been obtained using the data of a similar STAFF/DWP instrument onboard the Double Star TC-1 spacecraft (Santolík et al., 2005 b). Radial variation of the intensity of whistler-mode emissions was investigated for the $\mathrm{L}$ parameter ranging between 4 and 12. The chorus events showed an increased intensity of the wave magnetic field at $\mathrm{L}$ above 6 , consistent with intensifications of chorus which were previously observed by CLUSTER closer to the Earth at higher latitudes. This extends the radial distribution of chorus previously obtained by Meredith et al. (2003b) outward to higher L values into the region which GEOTAIL investigated (Nunn et al., 1997).

\section{Propagation of chorus from its source region}

Parrot et al. (2003b) and Parrot et al. (2004) reported observations with a CLUSTER spacecraft of intense chorus waves that propagated away from the equator, and, at the same time, observations with another space-shifted CLUSTER spacecraft of much lower-intensity waves at the same frequencies propagating toward the equator.

Using the observed wave normal directions of these waves, a backward ray tracing simulation predicted that the lowerintensity waves could have undergone the Lower Hybrid Resonance (LHR) reflection at low altitudes (Parrot et al., $2003 \mathrm{~b}$ ). The rays of these waves then pointed back to their anticipated source region close to the geomagnetic equator. This source region was, however, located at a different radial distance compared to the place of observation. The intensity ratio between magnetic component of the waves coming directly from the equator and waves returning to the equator was between 0.005 and 0.01 . The observations also showed that waves returning to the equator after the magnetospherical reflection still had a high degree of polarization, even if they started to lose the coherent structure of the chorus wave packets (Parrot et al., 2004). Contradictory results have been presented by Bortnik et al. (2006) who used a ray tracing simulation together with a model of the electron distribution functions, and showed that chorus originating at the magnetic equator with wave normal angles set at the generalized Gendrin angle can propagate only to latitudes of 10-20 degrees. Using these particular initial parameters, they found that chorus emissions are typically Landau damped before experiencing magnetospheric reflections.

On the other hand, Chum and Santolík (2005), Santolík et al. (2006), and Bortnik et al. (2007) showed that chorus can indeed magnetospherically reflect. They also showed that chorus can propagate from the equator to low altitudes toward the Earth if it is generated with Earthward inclined (downward) wave vectors. This result can be used to explain observations of low-altitude electromagnetic ELF hiss at subauroral latitudes. Santolík et al. (2006) reported observations of a divergent propagation pattern of these waves: they propagate with downward directed wave vectors which are slightly equatorward inclined at lower magnetic latitudes and slightly poleward inclined at higher latitudes. Reverse ray tracing with different plasma density models indicated a possible source near the geomagnetic equator at a radial distance between 5 and 7 Earth radii, and a generation mechanism acting on highly oblique wave vectors. Additionally, waveforms received at altitudes of $700-1200 \mathrm{~km}$ by the Freja and DEMETER spacecraft showed that low-altitude ELF hiss contains discrete frequency-time structures resembling wave packets of whistler mode chorus. Detailed measurements of the CLUSTER spacecraft indicated that the frequency-time structure and frequencies of chorus observed by CLUSTER along the reverse ray-paths of ELF hiss are consistent with the hypothesis that the ELF hiss is a low-altitude manifestation of whistler-mode chorus.

These ray paths have been also successfully simulated by the direct ray tracing method of Chum and Santolík (2005), for a source emitting with oblique and Earthward directed wave vectors. This paper also contains the first published description of a class of ray paths which penetrate from the chorus source region through the plasmapause density gradient into the plasmasphere (see Fig. 2). Importantly enough, Chum and Santolík (2005) also discovered that the wave-normal angles of these waves never get close to the whistler-mode resonance cone during their propagation from the source. This means that the wave refractive index stays low and that we cannot expect any important effect of Landau damping. Moreover, the wave normals are nearly fieldaligned when the waves again cross the equator inside the plasmasphere which is consistent with the observed wavenormals of plasmaspheric hiss and which makes possible further amplification of these waves (e.g. Solomon et al., 1988; Santolík et al., 2001).

As noted by Chum and Santolík (2005), and Santolík et al. (2006), Earthward propagating chorus can thus be considered as a possible additional candidate for the embryonic source of plasmaspheric hiss. Sonwalkar and Inan (1989), and recently Green et al. (2005) have presented evidence 


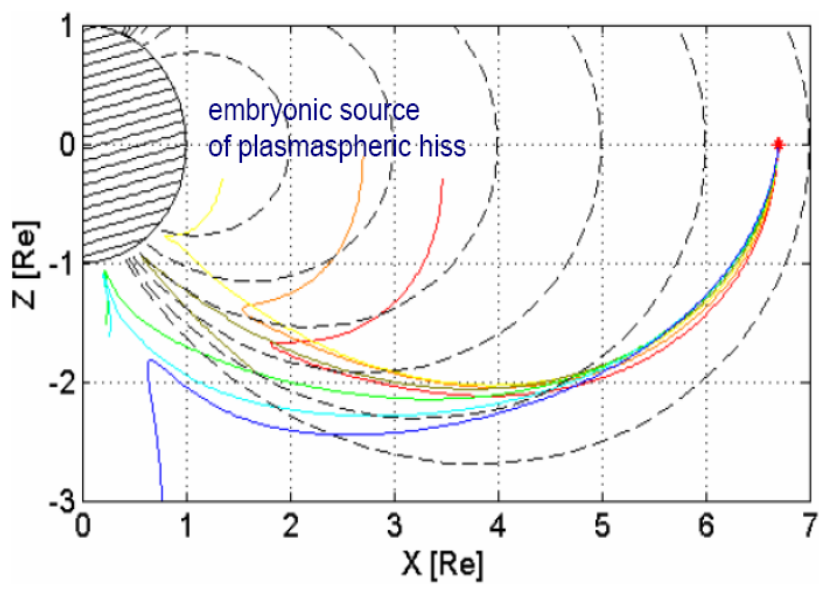

Fig. 2. Ray tracing simulation showing a class of ray paths which penetrate from the chorus source region at $X=6.6 R_{E}$ through the plasmapause density gradient into the plasmasphere, as it is indicated by red, yellow and orange lines (from Chum and Santolík, 2005).

on lightning-induced whistlers as the embryonic source of plasmaspheric hiss. Equatorward reflected ELF hiss which is most probably related to chorus emissions might represent another simultaneously acting embryonic source. Dependence of plasmaspheric hiss on the substorm activity, reported by Meredith et al. (2004), could be in favor of this chorus-related embryonic source. The original results of Chum and Santolík (2005) were reproduced and confirmed by a recent study of Bortnik et al. (2008) who obtained the same effect and who verified that there indeed is no substantial damping of waves during propagation from the chorus source into the plasmasphere.

\section{Comparison of observations with nonlinear theory}

The above described results of the CLUSTER project provide an important feedback for theories describing the chorus generation mechanism. Demekhov et al. (2003) compared the growth rates of whistler-mode wave packets obtained by the backward wave oscillator (BWO) theory (Trakhtengerts, 1995, 1999; Trakhtengerts and Rycroft, 2000) with observations of Santolík et al. (2003) and came to the conclusion that the observed range of growth rates $\left(34-420 \mathrm{~s}^{-1}\right)$ can be matched by the theory using reasonable fluxes of resonant electrons.

Properties of the observed chorus emissions are compared by Trakhtengerts et al. (2004) with the theoretical properties of the BWO mechanism. According to this theory, a succession of whistler wave packets is generated in a nearequatorial source region with temporal and spatial characteristics close to those observed by the four CLUSTER spacecraft. Amplitudes and frequency spectra of chorus, as well as dynamical features of the Poynting flux, are estimated and compared with the CLUSTER measurements. A good agreement is found for the observed and predicted lengths of the generation region measured along the magnetic field lines, characteristic periods of succession and global frequencytime slopes of chorus wave packets, growth rates of the substructure, and for saturation wave amplitudes. The nonlinear stage of chorus generation is determined by trapping of resonant energetic electrons that leads to formation of a sideband wave at a time scale between 6 and $60 \mathrm{~ms}$, as calculated for the observed plasma parameters. Therefore, a temporal modulation in wave amplitude can be expected, as confirmed by the results shown above in Sect. 3 .

Trakhtengerts et al. (2007) investigated frequencies of individual chorus wave packets close to the geomagnetic equator in the frame of the BWO theory. This model predicts that the frequency of chorus is determined by the parallel (with respect to the field line) velocity of a step-like deformation in the distribution function of resonant electrons. This velocity decreases during the generation of a chorus wave packet. At the same time, the electrons move through the generation region in the opposite direction to the wave group velocity. The highest frequencies of rising chorus wave packets are therefore generated by lower-energy resonant electrons which are leaving the source, but in the initial part of the wave path through the generation region. Lower frequencies should be consequently generated when the wave packets propagate to the other side of the chorus source. Since the wave packets can be generated with both signs of parallel component of the group velocity, the outer regions of the chorus source should contain both narrow-bandwidth wave packets at the highest frequencies and full-bandwidth packets, but only the higherfrequency parts of chorus wave packets should be observed well inside the source region. These predictions of the BWO theory have been successfully compared with the data of the WBD instrument onboard CLUSTER. The observations indicate that lower frequencies disappear when the spacecraft moved closer to the geomagnetic equator where we expect to find the average center of the source region. Santolík et al. (2008) related the frequencies of chorus wave packets to the central position of chorus source region obtained from the Poynting flux measurements onboard CLUSTER. The results confirmed the conclusions of Trakhtengerts et al. (2007).

The simulation study of Katoh and Omura (2006) tested the model of resonant currents formed by nonlinearly trapped resonant electrons by waves propagating in the inhomogeneous background magnetic field (Nunn, 1974). Katoh and Omura (2006) used the Electron Hybrid Model where the background cold electrons are treated as a fluid while energetic electrons are treated as relativistic particles. They succeeded to reproduce a coherent emission with a rising tone triggered by an intense constant frequency wave pulse with an amplitude of $\approx 5 \times 10^{-4} B_{0}$. Using a highly anisotropic loss cone distribution of resonant electrons, $\mathrm{Ka}$ toh and Omura (2007) were able to reproduce realistic chorus elements by the same simulation method but starting the 
simulation with the thermal noise, without using the strong coherent triggering signal. They succeeded to simulate wave packets with sweep rates of $\approx 9 \mathrm{kHz} / \mathrm{s}$, approximately corresponding to observations of Santolík et al. (2003) for similar plasma densities. The maximum reported linear growth rate of $230 \mathrm{~s}^{-1}$ and the simulated amplitudes of chorus wave packets $\left(10^{-4} B_{0} \approx 500 \mathrm{pT}\right)$ also correspond to the observations, as reported above in Sect. 3.

Nunn et al. (2008) $)^{1}$ present new results obtained using a Vlasov Hybrid Simulation (VHS) code to simulate the dynamical spectra of chorus. The results compare well to the observations of the CLUSTER mission. The simulation, following the theory of Nunn (1974), predicts the nonlinear resonant current, with a component in phase with the electric field that gives the power input to the wave field, and with a component in phase with the wave magnetic field that induces the sweeping frequency. The obtained sweep rates in the range $1-10 \mathrm{kHz} / \mathrm{s}$ well match the observations. The sweep rate is also, in agreement with observations, found to increase as the background plasma density decreases, leading to the conclusion that the nonlinear electron cyclotron resonance is the generation mechanism of chorus emissions.

\section{Conclusions}

During the last several years, emissions of whistler-mode chorus are receiving an increased attention. The main reason for this increased interest is connected with radiation belt physics and modeling, where investigation of chorus can play a role in understanding mechanisms of local acceleration of energetic electrons in the outer electron Van Allen radiation belts.

Due to this recent increase of publication activity, it is difficult to cover entirely the subject of new observational and theoretical results on whistler-mode chorus in a single review paper. Instead, the present review only summarizes selected results published during the last five years. Selection of the results discussed has been mainly based on the analysis of measurements of the CLUSTER spacecraft to show the results demonstrating the nonlinear nature of chorus emissions.

Although the experimental results are the main focus of this review, new theoretical and simulation results have also been extensively discussed here, mainly in connection with nonlinear effects on substructure and amplitudes of chorus wave packets. A special section has also been devoted to papers allowing comparison of chorus measurements with corresponding values deduced from nonlinear theory and simulations where new promising directions arise. However, many new results, mainly concerning the radiation belt dynamics, have been omitted or only briefly mentioned. This

\footnotetext{
${ }^{1}$ Nunn, D., Santolik, O., Rycroft, M., and Trakhtengerts, V.: On the numerical modelling of VLF chorus dynamical spectra, Ann. Geophys., submitted, 2008.
}

fast evolving domain of global modeling of radiation belt dynamics starts to show the need for solid data on both the properties and effects of whistler-mode chorus. Proper knowledge of these properties is hardly possible without investigations of the microphysics of nonlinear interactions of chorus waves with energetic electrons.

Besides the fact that many new results on chorus have recently appeared, there are still many unknowns in our understanding of source mechanisms and effects of this dynamical nonlinear phenomenon. In the near future, new developments of nonlinear theory and electromagnetic simulation techniques based on particle and Vlasov codes can be expected, as well as their continuing tests against experimental data. New experimental results can be expected in determining the propagation characteristics of chorus wave packets, further analysis of their amplitudes and fine structure, in experimental investigations of wave-particle interactions, and in systematic investigations based on the increasing volumes of experimental data on whistler-mode chorus emissions.

Acknowledgements. This work has been done with help of many fruitful discussions with colleagues, namely V. Y. Trakhtengerts (recently deceased) and A. G. Demekhov from IAP Nizhny Novgorod, Russia; D. A. Gurnett, J. S. Pickett, C. A. Kletzing, and A. Breneman at the University of Iowa, Iowa City, USA; D. Nunn from the University of Southampton, UK; Y. Omura from Kyoto University, Japan; M. Parrot and P. Décréau from LPCE/CNRS, Orléans, France; N. Cornilleau-Wehrlin and P. Canu from CETP/IPSL, Vélizy, France; E. E. Titova and B. V. Kozelov from PGI Apatity, Russia; U. S. Inan and T. F. Bell from Stanford University, USA; J. Chum and F. Jiricek at IAP Prague, and others. Special thanks are due to M. J. Rycroft for encouraging discussions and careful corrections. This research has made use of NASA's Astrophysics Data System. The Cluster WBD measurements were supported by the NASA grant NNX07AI24G. This work was supported under the contracts ESA PECS No. 98025, ME 842, and GAAV IAA 301120601.

Edited by: K. Stasiewicz

Reviewed by: M. J. Rycroft and another anonymous referee

\section{References}

Albert, J. M.: Efficient approximations of quasi-linear diffusion coefficients in the radiation belts, J. Geophys. Res., 113, A06208, doi:10.1029/2007JA012936, 2008.

Anderson, R. R. and Maeda, K.: VLF emissions associated with enhanced magnetospheric electrons, J. Geophys. Res., 82, 135146, 1977.

Andronov, A. A. and Trakhtengerts, V. Y.: Kinetic instability of the Earth's outer radiation belt, Geomagnetism and Aeronomy, 4, 233-242, 1964.

Bell, T. F.: The nonlinear gyroresonance interaction between energetic electrons and coherent VLF waves propagating at an arbitrary angle with respect to the earth's magnetic field, J. Geophys. Res., 89, 905-918, 1984.

Bell, T. F.: The wave magnetic field amplitude threshold for nonlinear trapping of energetic gyroresonant and Landau resonant 
electrons by nonducted VLF waves in the magnetosphere, J. Geophys. Res., 91, 4365-4379, 1986.

Bortnik, J., Inan, U. S., and Bell, T. F.: Landau damping and resultant unidirectional propagation of chorus waves, Geophys. Res. Lett., 33, L03102, doi:10.1029/2005GL024553, 2006.

Bortnik, J., Thorne, R. M., Meredith, N. P., and Santolik, O.: Ray tracing of penetrating chorus and its implications for the radiation belts, Geophys. Res. Lett., 34, L15109, doi:10.1029/2007GL030040, 2007.

Bortnik, J., Thorne, R. M., and Meredith, N. P.: The unexpected origin of plasmaspheric hiss from discrete chorus emissions, Nature, 452, 62-66, doi:10.1038/nature06741, 2008.

Breneman, A. W., Kletzing, C. A., Chum, J., and Santolik, O.: Multi-Spacecraft Observations of Chorus Dispersion and Source Location, J. Geophys. Res., 112, A05221, doi:10.1029/2006JA012058, 2007.

Burtis, W. J. and Helliwell, R. A.: Magnetospheric chorus: Occurrence patterens and normalized frequency, Planet. Space Sci., 24, 1007-1024, 1976.

Burton, R. K.: Critical electron pitch angle anisotropy necessary for chorus generation, J. Geophys. Res., 81, 4779-4781, 1976.

Burton, R. K. and Holzer, R. E.: The origin and propagation of chorus in the outer magnetosphere, J. Geophys. Res., 79, 1014 1023, 1974

Cattell, C., Wygant, J. R., Goetz, K., Kersten, K., Kellogg, P. J., von Rosenvinge, T., Bale, S. D., Roth, I., Temerin, M., Hudson, M. K., Mewaldt, R. A., Wiedenbeck, M., Maksimovic, M., Ergun, R., Acuna, M., and Russell, C. T.: Discovery of very large amplitude whistler-mode waves in Earths radiation belts, Geophys. Res. Lett., 35, L01105, doi:10.1029/2007GL032009, 2008.

Chen, Y., Reeves, G. D., and Friedel, R. H. W.: The energization of relativistic electrons in the outer Van Allen radiation belt, Nature Phys., 3, 614-617, 2007.

Chum, J. and Santolík, O.: Propagation of whistler-mode chorus to low altitudes: divergent ray trajectories and ground accessibility, Ann. Geophys., 23, 3727-3738, 2005,

http://www.ann-geophys.net/23/3727/2005/.

Chum, J., Santolik, O., Breneman, A. W., Kletzing, C. A., Gurnett, D. A., and Pickett, J. S.: Chorus source properties that produce time shifts and frequency range differences observed on different Cluster spacecraft, J. Geophys. Res., 112, A06206, doi:10.1029/2006JA012061, 2007.

Cornilleau-Wehrlin, N., Etcheto, J., and Burton, R. K.: Detailed analysis of magnetospheric ELF chorus: Preliminary results, J. Atmos. Terr. Phys., 38, 1201-1210, 1976.

Cornilleau-Wehrlin, N., Gendrin, R., Lefeuvre, F., Parrot, M., Grard, R., Jones, D., Bahnsen, A., Ungstrup, E., and Gibbons, W.: VLF electromagnetic waves observed onboard GEOS-1, Space Sci. Rev., 22, 371-382, 1978.

Cornilleau-Wehrlin, N., Chanteur, G., Perraut, S., Rezeau, L., Robert, P., Roux, A., Villedary, C. D., Canu, P., Maksimovic, M., Conchy, Y. D., Hubert, D., Lacombe, C., Lefeuvre, F., Parrot, M., Pincon, J.-L., Decrau, P., Harvey, C. C., Louarn, P., Santolik, O., Alleyne, H. S. C., Roth, M., et al.: First results obtained by the Cluster STAFF experiment, Ann. Geophys., 21, 437-456, 2003, http://www.ann-geophys.net/21/437/2003/.

Décréau, P. M. E., Fergeau, P., Krasnoselskikh, V., Guirriec, E. L., Lévêque, M., Martin, P., Randriamboarison, O., Rauch, J. L.,
Sené, F. X., Séran, H. C., Trotignon, J. G., Canu, P., Cornilleau, N., de Féraudy, H., Alleyne, H., Yearby, K., Mögensen, P. B., Gustafsson, G., André, M., Gurnett, D. A., Darrouzet, F., Lemaire, J., Harvey, C. C., Travnicek, P., et al.: Early results from the Whisper instrument on Cluster: an overview, Ann. Geophys., 19, 1241-1258, 2001,

http://www.ann-geophys.net/19/1241/2001/.

Demekhov, A. G., Nunn, D., and Trakhtengerts, V. Y.: Backward wave oscillator regime of the whistler cyclotron instability in an inhomogeneous magnetic field, Phys. Plasmas, 10, 4472-4477, doi:10.1063/1.1620507, 2003.

Demekhov, A. G., Trakhtengerts, V. Y., Rycroft, M. J., and Nunn, D.: Electron acceleration in the magnetosphere by whistlermode waves of varying frequency, Geomag. Aeron., 46, 711716, doi:10.1134/S0016793206060053, 2006.

Escoubet, C. P., Schmidt, R., and Goldstein, M. L.: Cluster - Science and Mission Overview, Space Sci. Rev., 79, 11-32, 1997.

Goldstein, B. E. and Tsurutani, B. T.: Wave normal directions of chorus near the equatorial source region, J. Geophys. Res., 89, 2789-2810, 1984.

Green, J. L., Boardsen, S., Garcia, L., Taylor, W. W. L., Fung, S. F., and Reinisch, B. W.: On the origin of whistler mode radiation in the plasmasphere, J. Geophys. Res., 110, A03201, doi:10.1029/2004JA010495, 2005.

Gurnett, D. A. and O'Brien, B. J.: High-latitude geophysical studies with satellite Injun 3, 5. Very-low-frequency electromagnetic radiation, J. Geophys. Res., 69, 65-89, 1964.

Gurnett, D. A., Huff, R. L., and Kirchner, D. L.: The Wide-Band Plasma Wave Investigation, Space Sci. Rev., 79, 195-208, 1997.

Gurnett, D. A., Huff, R. L., Pickett, J. S., Persoon, A. M., Mutel, R. L., Christopher, I. W., Kletzing, C. A., Inan, U. S., Martin, W. L., Bougeret, J.-L., Alleyne, H. S. C., and Yearby, K. H.: First results from the Cluster wideband plasma wave investigation, Ann. Geophys., 19, 1259-1272, 2001, http://www.ann-geophys.net/19/1259/2001/.

Helliwell, R. A.: A theory of discrete emissions from the magnetosphere, J. Geophys. Res., 72, 4773-4790, 1967.

Helliwell, R. A.: Low-frequency waves in the magnetosphere, Rev. Geophys., 7, 281-303, 1969.

Horne, R. B.: Acceleration of killer electrons, Nature Phys., 3, 590591, 2007.

Horne, R. B. and Thorne, R. M.: Potential waves for relativistic electron scattering and stochastic acceleration during magnetic storms, Geophys. Res. Lett., 25, 3011-3014, 1998.

Horne, R. B. and Thorne, R. M.: Relativistic electron acceleration and precipitation during resonant interactions with whistler-mode chorus, Geophys. Res. Lett., 30, 1527, doi:10.1029/2003GL016973, 2003.

Horne, R. B., Glauert, S. A., and Thorne, R. M.: Resonant diffusion of radiation belt electrons by whistler-mode chorus, Geophys. Res. Lett., 30, 1493, doi:10.1029/2003GL016963, 2003.

Horne, R. B., Thorne, R. M., Shprits, Y. Y., Meredith, N. P., Glauert, S. A., Smith, A. J., Kanekal, S. G., Baker, D. N., Engebretson, M. J., Posch, J. L., Spasojevic, M., Inan, U. S., Pickett, J. S., and Decreau, P. M. E.: Wave acceleration of electrons in the Van Allen radiation belts, Nature, 437, 227-230, doi:10.1038/nature03939, 2005.

Inan, U. S., Chiu, Y. T., and Davidson, G. T.: Whistler-mode chorus and morningside aurorae, Geophys. Res. Lett., 19, 653-656, 
1992.

Inan, U. S., Platino, M., Bell, T. F., Gurnett, D. A., and Pickett, J. S.: Cluster measurements of rapidly moving sources of ELF/VLF chorus, J. Geophys. Res., 109, A05214, doi:10.1029/2003JA010289, 2004.

Katoh, Y. and Omura, Y.: A study of generation mechanism of VLF triggered emission by self-consistent particle code, J. Geophys. Res., 111, A12207, doi:10.1029/2006JA011704, 2006.

Katoh, Y. and Omura, Y.: Computer simulation of chorus wave generation in the Earths inner magnetosphere, Geophys. Res. Lett., 34, L03102, doi:10.1029/2006GL028594, 2007.

Kennel, C. F. and Petschek, H. E.: Limit on stable trapped particle fluxes, J. Geophys. Res., 71, 1-28, 1966.

Koons, H. C. and Roeder, J. L.: A survey of equatorial magnetospheric wave activity between 5 and $8 R_{E}$, Planet. Space. Sci., 38, 1335-1341, 1990.

Lauben, D. S., Inan, U. S., Bell, T. F., Kirchner, D. L., Hospodarsky, G. B., and Pickett, J. S.: VLF chorus emissions observed by Polar during the January 10, 1997 magnetic cloud, Geophys. Res. Lett., 25, 2995-2998, 1998.

Lauben, D. S., Inan, U. S., Bell, T. F., and Gurnett, D. A.: Source characteristics of ELF/VLF chorus, J. Geophys. Res., 107, 1429, doi:10.1029/2000JA003019, 2002.

LeDocq, M. J., Gurnett, D. A., and Hospodarsky, G. B.: Chorus source locations from VLF Poynting flux measurements with the Polar spacecraft, Geophys. Res. Lett., 25, 4063-4066, 1998.

Meredith, N. P., Horne, R. B., and Anderson, R. R.: Substorm dependence of chorus amplitudes: Implications for the acceleration of electrons to relativistic energies, J. Geophys. Res., 106, 13 165-13 178, 2001.

Meredith, N. P., Horne, R. B., Summers, D., Thorne, R. M., Iles, R. H. A., Heynderickx, D., and Anderson, R. R.: Evidence for acceleration of outer zone electrons to relativistic energies by whistler mode chorus, Ann. Geophys., 20, 967-979, 2002, http://www.ann-geophys.net/20/967/2002/.

Meredith, N. P., Cain, M., Horne, R. B., Thorne, R. M., Summers, D., and Anderson, R. R.: Evidence for chorus-driven electron acceleration to relativistic energies from a survey of geomagnetically disturbed periods, J. Geophys. Res., 108, 1248, doi:10.1029/2002JA009764, 2003a.

Meredith, N. P., Horne, R. B., Thorne, R. M., and Anderson, R. R.: Favored regions for chorus-driven electron acceleration to relativistic energies in the Earth's outer radiation belt, Geophys. Res. Lett., 30, 1871, doi:10.1029/2003GL017698, 2003 b.

Meredith, N. P., Horne, R. B., Thorne, R. M., Summers, D., and Anderson, R. R.: Substorm dependence of plasmaspheric hiss, J. Geophys. Res., 109, A06209, doi:10.1029/2004JA010387, 2004.

Nunn, D.: A self-consistent theory of triggered VLF emissions, Planet. Space Sci., 22, 349-378, 1974.

Nunn, D., Omura, Y., Matsumoto, H., Nagano, I., and Yagitani, S.: The numerical simulation of VLF chorus and discrete emissions observed on the Geotail satellite using a Vlasov code, J. Geophys. Res., 102, 27 083-27 097, 1997.

Nunn, D., Demekhov, A., Trakhtengerts, V., and Rycroft, M. J.: VLF emission triggering by a highly anisotropic electron plasma, Ann. Geophys., 21, 481-492, 2003, http://www.ann-geophys.net/21/481/2003/.

Nunn, D., Rycroft, M. J., and Trakhtengerts, V.: A parametric study of the numerical simulations of triggered VLF emissions, Ann.
Geophys., 23, 3655-3666, 2005,

http://www.ann-geophys.net/23/3655/2005/.

Omura, Y. and Summers, D.: Computer simulations of relativistic whistler-mode wave-particle interactions, Phys. Plasmas, 11, 3530-3534, 2004.

Omura, Y. and Summers, D.: Dynamics of high-energy electrons interacting with whistler mode chorus emissions in the magnetosphere, J. Geophys. Res., 111, A09222, doi:10.1029/2006JA011600, 2006.

Omura, Y., Nunn, D., Matsumoto, H., and Rycroft, M. J.: A review of observational, theoretical and numerical studies of VLF triggered emissions, J. Atmos. Terr. Phys., 53, 351-368, 1991.

Omura, Y., Furuya, N., and Summers, D.: Relativistic turning acceleration of resonant electrons by coherent whistler mode waves in a dipole magnetic field, J. Geophys. Res., 112, A06236, doi:10.1029/2006JA012243, 2007.

Parrot, M., Santolík, O., Cornilleau-Wehrlin, N., Maksimovic, M., and Harvey, C.: Source location of chorus emissions observed by Cluster, Ann. Geophys., 21, 473-480, 2003a,

http://www.ann-geophys.net/21/473/2003/.

Parrot, M., Santolík, O., Cornilleau-Wehrlin, N., Maksimovic, M., and Harvey, C.: Magnetospherically reflected chorus waves revealed by ray tracing with CLUSTER data, Ann. Geophys., 21, 1111-1120, 2003b, http://www.ann-geophys.net/21/1111/2003/.

Parrot, M., Santolík, O., Gurnett, D., Pickett, J., and CornilleauWehrlin, N.: Characteristics of magnetospherically reflected chorus waves observed by CLUSTER, Ann. Geophys., 22, 25972606, 2004, http://www.ann-geophys.net/22/2597/2004/.

Platino, M., Inan, U. S., Bell, T. F., Pickett, J. S., and Canu, P.: Rapidly moving sources of upper band ELF/VLF chorus near the magnetic equator, J. Geophys. Res., 111, 9218, doi:10.1029/2005JA011468, 2006.

Santolík, O. and Gurnett, D. A.: Transverse dimensions of chorus in the source region, Geophys. Res. Lett., 30, 1031, doi:10.1029/2002GL016178, 2003.

Santolík, O., Parrot, M., Storey, L., Pickett, J., and Gurnett, D. A.: Propagation analysis of plasmaspheric hiss using Polar PWI measurements, Geophys. Res. Lett., 28, 1127-1130, 2001.

Santolík, O., Gurnett, D. A., Pickett, J. S., Parrot, M., and Cornilleau-Wehrlin, N.: Spatio-temporal structure of storm-time chorus, J. Geophys. Res., 108, 1278, doi:10.1029/2002JA009791, 2003.

Santolík, O., Gurnett, D. A., and Pickett, J. S.: Multipoint investigation of the source region of storm-time chorus, Ann. Geophys., 22, 2555-2563, 2004a,

http://www.ann-geophys.net/22/2555/2004/.

Santolík, O., Gurnett, D. A., Pickett, J. S., Parrot, M., and Cornilleau-Wehrlin, N.: A microscopic and nanoscopic view of storm-time chorus on 31 March 2001, Geophys. Res. Lett., 31, L02801, doi:10.1029/2003GL018757, 2004b.

Santolík, O., Gurnett, D. A., Pickett, J. S., Parrot, M., and Cornilleau-Wehrlin, N.: Central position of the source region of storm-time chorus, Planet. Space Sci., 53, 299-305, 2005 a.

Santolík, O., Macusova, E., Yearby, K. H., Cornilleau-Wehrlin, N., and Alleyne, H. S. K.: Radial variation of whistler-mode chorus: First results from the STAFF/DWP instrument onboard the Double Star TC 1 spacecraft, Ann. Geophys., 23, 2937-2942, 2005b, 
http://www.ann-geophys.net/23/2937/2005/.

Santolík, O., Chum, J., Parrot, M., Gurnett, D. A., Pickett, J. S., and Cornilleau-Wehrlin, N.: Propagation of whistler mode chorus to low altitudes: Spacecraft observations of structured ELF hiss, J. Geophys. Res., 111, A10208, doi:10.1029/2005JA011462, 2006.

Santolík, O., Macusova, E., Titova, E. E., Kozelov, B. V., Gurnett, D. A., Picket, J. S., Trakhtengerts, V. Y., and Demekhov, A. G.: Frequencies of wave packets of whistler-mode chorus inside its source region: a case study, Ann. Geophys., 26, 16651670, 2008, http://www.ann-geophys.net/26/1665/2008/.

Sazhin, S. S. and Hayakawa, M.: Magnetospheric chorus emissions: A review, Planet. Space Sci., 40, 681-697, 1992.

Shprits, Y. Y., Thorne, R. M., Horne, R. B., and Summers, D.: Bounce-averaged diffusion coefficients for fieldaligned chorus waves, J. Geophys. Res., 111, A10225, doi:10.1029/2006JA011725, 2006.

Shprits, Y. Y., Meredith, N. P., and Thorne, R. M.: Parameterization of radiation belt electron loss timescales due to interactions with chorus waves, Geophys. Res. Lett., 34, L11110, doi:10.1029/2006GL029050, 2007.

Solomon, J., Cornilleau-Wehrlin, N., Korth, A., and Kremser, G.: An experimental study of ELF/VLF hiss generation in the Earth's magnetosphere, J. Geophys. Res., 93, 1839-1847, 1988.

Sonwalkar, V. S. and Inan, U. S.: Lightning as an embryonic source of VLF hiss, J. Geophys. Res., 94, 6986-6994, 1989.

Storey, L. R. O.: An investigation of whistling atmospherics, Phil. Trans. Roy. Soc. London, A246, 113-141, 1953.

Summers, D., Thorne, R. M., and Xiao, F.: Relativistic theory of waveparticle resonant diffusion with application to electron acceleration in the magnetosphere, J. Geophys. Res., 103, $20487-$ $20500,1998$.
Summers, D., Ni, B., and Meredith, N. P.: Timescales for radiation belt electron acceleration and loss due to resonant wave-particle interactions: 2. Evaluation for VLF chorus, ELF hiss, and electromagnetic ion cyclotron waves, J. Geophys. Res., 112, A04207, doi:10.1029/2006JA011993, 2007a.

Summers, D., Ni, B., and Meredith, N. P.: Timescales for radiation belt electron acceleration and loss due to resonant waveparticle interactions: 1. Theory, J. Geophys. Res., 112, A04206, doi:10.1029/2006JA011801, 2007b.

Trakhtengerts, V.: Magnetosphere cyclotron maser: backward wave oscillator generation regime, J. Geophys. Res., 100, 17205 $17210,1995$.

Trakhtengerts, V.: A generation mechanism for chorus emission, Ann. Geophys., 17, 95-100, 1999, http://www.ann-geophys.net/17/95/1999/.

Trakhtengerts, V. and Rycroft, M.: Whistler electron interactions in the magnetosphere: new results and novel approaches, J. Atmos. Sol.-Terr. Phys., 62, 1719-1733, 2000.

Trakhtengerts, V. Y., Demekhov, A. G., Titova, E. E., Kozelov, B. V., Santolik, O., Gurnett, D., and Parrot, M.: Interpretation of Cluster data on chorus emissions using the backward wave oscillator model, Phys. Plasmas, 11, 1345-1351, 2004.

Trakhtengerts, V. Y., Demekhov, A. G., Titova, E. E., Kozelov, B. V., Santolik, O., Macusova, E., Gurnett, D. A., Pickett, J. S., Rycroft, M. J., and Nunn, D.: Formation of VLF chorus frequency spectrum: Cluster data and comparison with the backward wave oscillator model, Geophys. Res. Lett., 34, L02104, doi:10.1029/2006GL027953, 2007.

Tsurutani, B. T. and Smith, E. J.: Postmidnight chorus: a substorm phenomenon, J. Geophys. Res., 79, 118-127, 1974.

Tsurutani, B. T. and Smith, E. J.: Two types of magnetospheric ELF chorus and their substorm dependence, J. Geophys. Res., 82, 5112-5128, 1977. 